\title{
RESEARCH
}

\section{An Exploratory Mixed Methods Study of Standardized Patient Comments on Empathy and Student Communication Scores}

\author{
Logan T. Murry, PharmD, Jeffrey C. Reist, PharmD, Michelle A. Fravel, PharmD, Laura E. Knockel, PharmD, \\ Mathew J. Witry, $\mathrm{PhD}$
}

The University of Iowa, College of Pharmacy, Iowa City, Iowa

Corresponding Author: Logan T. Murry, The University of Iowa, College of Pharmacy, 180 S. Grand Ave., Iowa City, IA 52242.
Tel: 319-325-9055. Email: logan.murry@ @mail.com

Submitted March 16, 2021; accepted June 15, 2021; ePublished June 2021

Objective. The objectives of this study were to 1) quantify students' communication ability using standardized patient (SP) communication rubric scores, 2) describe and categorize SP comments of student empathy and 3) test the relationship between communication scores and empathy.

Methods. A concurrent mixed methods research design was used to assess a graded Performance Based Assessment (PBA) of student pharmacists at a college of pharmacy in the Midwest US. PBA rubrics $(n=218)$ completed by SPs contained 20 assessment items with space for open-ended feedback. Scoring categories for communication assessment included: Yes, Inconsistent, No, and N/A. Descriptive statistics were calculated for rubric scores. SP feedback was transformed into categorical data by categorizing assessments suggesting high empathy, mixed empathy, and low empathy encounters. Kruskal-Wallis ANOVA was used to test the relationship between empathy category and communication score.

Results. SPs wrote comments on 141 of 218 rubrics (64.7\%). The mean communication score was $38.96 \pm 1.64$ (Range of 31 to 40) out of a maximum 40 points. The totals for the low, mixed, and high empathy category transformations were 6 (4.3\%), 95 (67.4\%), and 40 (28.4\%), respectively. The results of the Kruskal-Wallis ANOVA were statistically significant, suggesting that communication scores were different between empathy categories.

Conclusion. There is a positive association between scores on communication rubrics and student empathy categorization, with students exhibiting different levels of clinical empathy. While the PBA of interest was not specifically focused on empathy, SPs frequently provided empathy feedback to students suggesting empathy was important to the encounter.

Keywords: empathy, communication, clinical empathy, performance-based assessments, standardized patients

\section{INTRODUCTION}

Empathy is a critical component of patient-centered communication, professional development, and pharmacy education and practice. ${ }^{1-6}$ Empathy, as it applies to health professionals, can be described as a cognitive attribute that involves an understanding of a patient's concerns, the capacity to communicate this understanding, and an intention to help. ${ }^{5,7,8}$ The concept of clinical empathy is useful for describing dialogue in the patient-provider clinical relationships. ${ }^{9}$

${ }^{10}$ Clinical empathy requires the provider to 1) understand the patient's perspectives, feelings, and meaning of their clinical situation; 2) communicate their understanding and check its accuracy; and 3) act on that understanding, with the patient, in a helpful and therapeutic way. ${ }^{9,11-13}$ Empathy, when demonstrated in clinical encounters, has been shown to improve the patient experience, increase adherence to treatment recommendations, improve health outcomes, and enhance trust. ${ }^{14-18}$ In addition to the positive effects on patient experiences and care, empathy also has been shown to provide personal benefits to providers, with evidence that providers who engage in empathetic communication experience greater professional fulfillment and decreased feelings of burnout. ${ }^{9}{ }^{19}$ With a number of implications to pharmacy practice, patient care, and provider well-being, pharmacists and student pharmacists stand to benefit from education and training focused on empathetic communication and clinical empathy. As such, empathy and empathetic communication have been included as a required component of accreditation standards related to providing patient-centered care and are skills that can be taught to health professionals. ${ }^{2,7,20}$

To determine the effectiveness of empathy training for professional students, multiple studies have used crosssectional assessments, with emphasis on evaluating educational interventions to increase the use of empathetic patient- 
centered communication while performing patient consultations. ${ }^{20,21}$ Recent studies have focused on assessing and cultivating student pharmacist empathy. ${ }^{22-25}$ One way of cultivating empathy in health care professional students is through role-playing activities, frequently employing standardized patients (SPs) to engage with students in simulated clinical encounters and assess communication abilities. ${ }^{26}$. The focus of a SP in these encounters is not unlike that of an actual patient, making the SP well-equipped to provide valuable feedback that will assist students in their empathy and communication development. ${ }^{27}$ Experiences with SPs are an essential component of comprehensive empathy assessment and may act as an educational intervention to improve empathy and self-reflection while measuring empathetic communication more objectively than self-assessment measures alone. ${ }^{28-30}$

With increased emphasis on facilitating student acquisition of empathetic communication skills, it is important to note that, empathy and empathetic communication skills decrease as individuals receive additional clinical education and experience. ${ }^{31,32}$ Given the importance of clinical empathy and the use of SPs to facilitate the acquisition of and assess empathetic communication skills, exploring how SPs evaluate students on communication and empathy using existing communication assessment rubrics may provide additional insight on the importance, acquisition, and exhibition of clinical empathy within simulated clinical encounters.

\section{OBJECTIVE}

The objectives of this study were to 1) quantify students' communication ability using SP communication rubric scores, 2) describe and categorize SP comments of student empathy and 3) test the relationship between quantitative rubric scores and empathy categorization using an integrative analysis of transformed qualitative comments.

\section{METHODS}

This was a retrospective evaluation of a graded Performance-Based Assessment (PBA) using SPs at a MidwestU.S. located college of pharmacy using a convergent mixed methods research design. The rationale for using mixed methods in this study was to expand and transform qualitative findings to understand how SP empathy perceptions influenced student communication scores and to explore the varying degrees to which students engaged in empathetic communication. A visual depiction of the mixed methods study design is included in Figure 1. The study was conducted in accordance with institutional IRB policies and was granted an exemption as non-human subject research.

The PBA scenario focused on a patient with several medications for hypertension and diabetes who was struggling with medication adherence. Students were tasked with obtaining information related to the medication regimen and difficulties with medications or disease state management. The primary objective of the PBA was to identify patient medication concerns and address medication non-adherence. SPs were asked to evaluate students on clinical and communication skills using a rubric developed and recently finalized by experts at six schools and colleges of pharmacy. ${ }^{33}$ Details on the specific development processes can be found elsewhere ${ }^{33}$, with efforts to further explore validity and reliability currently underway. SPs provided item scores and written feedback on communication and clinical rubrics as well as oral feedback immediately following the completion of the PBA encounter. SPs were not notified that written feedback would be used for an assessment of student empathy, minimizing potential bias. SPs are trained to assess clinical and communication scores at the university's college of medicine. PBA communication rubrics scored by SPs from two cohorts of third-year student pharmacists (218 students) were included in the evaluation, as these individuals were entering their Advanced Pharmacy Practice Experience (APPEs) rotations, and this was their last PBA using SPs. The two cohorts were similar, with all students completing comparable didactic and experiential training prior to the final PBA. Student pharmacist participants were deidentified by one of the course instructors before the analysis. Additionally, the clinical scenario and rubric used for assessment was identical for both cohorts. While all SPs completed assessment rubrics, only rubrics for students who received supplementary hand-written comments were included for analysis.

\section{Mixed Methods Study Design and Analysis}

For the quantitative data, total student communication scores were calculated from scores for 20 communication items across 6 domains: Initiating the Encounter (3 items), Establishing Trust and Respect (4 items), Using Verbal Communication Skills (5 items), Using Non-Verbal Communication Skills (2 items), Managing the Encounter (3 items), and Concluding the Encounter (3 items). While the original rubric was out of 20 points, all items were transformed into whole numbers while maintaining comparable differences across all items to facilitate analysis. As such, No maintained a zero-point value, Inconsistent was associated with a one-point value (increased from one-half point value), and Yes was associated with a two-point value (increased from one-point value). Non-applicable items (N/A) were also associated with a two-point value. As a result, the maximum obtainable score on communication assessment was 40 points. One item on the communication assessment rubric evaluates student empathy during the encounter; however, SPs were instructed to 
select N/A for student empathy as the encounter was not specifically focused on assessing empathy and SPs have historically struggled to interpret and score the empathy item. Descriptive statistics and frequencies were used to evaluate communication rubric scores for all students. For the qualitative analysis, open-ended comments left by SPs providing communication feedback were uploaded into the data management software MAXQDA 2020 (VERBI Software, 2019). Two of the study authors, a graduate student and associate professor in the department of Pharmacy Practice and Science not involved with the development or execution of the PBAs, independently performed a basic interpretive analysis on written feedback. These two study authors independently coded text segments for all rubrics included in the analysis and met to discuss initial codes and specific terminology associated with SP comments related to student pharmacist empathy.

To integrate the two datasets, a second round of deductive coding was performed by the same study authors involved in the initial coding. The deductive coding was applied to the entirety of the SP open-ended comments for each student to categorize each student into 1 of 3 empathy categories (Low Empathy, Mixed Empathy, and High Empathy). For each student, the empathy category was applied based on the prevalence and general affect of empathy-related terminology in the open-ended comments left by the SP. Student rubrics were categorized as "Low Empathy" when the majority of the comments reflected encounters where the SP reported the student missed opportunities to address their needs and/or communicated with them unempathetically (eg, I was interrupted... when you asked about how I was doing with diet and exercise, I said I walked $3 x$ week, and diet is going ok, but I am not interested in doing more about diet at this point. Even so, you said I should keep working on the diet). Student rubrics were categorized as "High Empathy" when students exclusively received positive feedback related to their ability to exhibit empathetic communication skills throughout the encounter (eg, Student displayed strong concern for my well-being...I felt very engaged, no judging...). Student rubrics were categorized as "Mixed Empathy" when SPs gave students both positive and negative feedback related to empathetic communication (eg, ...we spoke about different ways to elicit concerns from a patient and I am sure they will be able to do so in the future) or when empathy-related terminology was not explicitly or implicitly mentioned in the open-ended comments (eg,...not rushed, I think there is room to slow down a bit. It will help give you time to think.). A subset of empathy categories and representative quotes were checked by three clinical pharmacy educators involved in PBA development for accuracy and appropriate empathy categorization. The clinical pharmacy educators were presented with a sample of uncoded text provided by SPs and were asked to independently code the text into the aforementioned empathy categories, ensuring that the initial coding was an accurate assessment of student empathy category from the perspective of those immediately involved in clinical skill education and evaluation.

To test the relationship between rubric scores and empathy categorization for the integrated analysis, mean rank communication rubric scores were compared using Kruskal-Wallis ANOVA in IBM SPSS Statistics for Windows, Version 25 (IBM Corp., Armonk, N.Y., USA). Post-hoc analysis was performed with Dunn's pairwise tests performed on all three pairs of empathy categorization to identify significant differences in communication scores between empathy categories.

\section{RESULTS}

In total 141 of 218 rubrics $(64.7 \%)$ were included for analysis. Written, open-ended feedback was not available for seventy-seven rubrics, limiting the ability to assign empathy categories. From the 141 rubrics, the average communication score for student pharmacists was $38.96 \pm 1.64$, with a low score of 31 and a high of 40.

SPs provided a range of feedback on student communication. SPs frequently commented on nonverbal communication ability and what emotions or feelings the SP had during the encounter. Nearly all students were given at least one positive comment and most students were provided feedback on areas for improvement. Most SP feedback focused on students missing cues or opportunities to connect and communicate following the SP expressing a concern or need. Similarly, some students received feedback that the proposed clinical plan failed to consider patient needs and preferences. In the following SP comments, specific words are underlined to emphasize the negative experiences with the associated empathy code included in brackets following the statement.

You did not ask why I came in today, instead you started with your agenda. I was interrupted...I am not interested in doing more about diet at this point. Even so, you said I should keep working on the diet. [Low Empathy Comment]

The majority of SP comments indicated that students exhibited both high and low empathy in the encounters. Common deficiencies included SPs feeling as though students were rushing encounters, speaking over them, and struggled to elicit their concerns. SPs sometimes noted these deficiencies only occasionally happened within each encounter, with SPs reporting general confidence that students could correct these communication errors moving forward. 
There were a couple of times they spoke over me when explaining my medications... [Mixed Empathy Comment]

...we spoke about different ways to elicit concerns from a patient and I am sure they will be able to do so in the future. [Mixed Empathy Comment]

Finally, some SP comments suggested they experienced exclusively positive encounters. Positive SP feedback often focused on encounters where they felt heard, supported, and did not feel judged. High-empathy comments frequently resulted from student pharmacists addressing patient concerns and helping the patient develop a plan to address them.

[They] have a quiet confidence and openness about [them] that made me feel like I could trust [them] and talk
openly about my prescriptions and lifestyle changes. [High Empathy Comment]

I felt supported and encouraged throughout our conversation. [High Empathy Comment]

I appreciated the nonjudgmental attitude conveyed about my missing doses. I could have felt embarrassed but instead you told me I could take it in the mornings w/ my other meds, if that would help, which was a relief. When you told me I was doing a great job with the diet and exercise, I felt inspired to keep it up. [High Empathy Comment]

\section{Integration: Transformative Empathy Categorization and Analysis}

Of the 141 included rubrics, $6(4.3 \%)$ rubrics were categorized as low-empathy encounters, 95 (67.4\%) were categorized as mixed, and $40(28.4 \%)$ were categorized as exhibiting high-empathy during the encounter. Representative quotes for each of the three empathy categories are included in Table 1.

The results from the Kruskal-Wallis test showed a statistically significant difference in communication score between at least one pair of empathy categories, (Kruskal-Wallis $\mathrm{H}=24.621$, $\mathrm{df}=2$, $p$-value <.05), with a mean rank communication score of 12.08 for the Low Empathy category, 67.26 for the Mixed Empathy category, and 88.73 for the High Empathy category. Dunn's pairwise tests identified statistically significant differences in communication scores between Low Empathy and High Empathy categories, Low Empathy and Mixed Empathy categories, and High Empathy and Mixed Empathy categories ( $p$-values <.05, adjusted using Bonferroni correction). Descriptive statistics for empathy category and communication scores can be found in Table 2 .

\section{DISCUSSION}

Overall, students received high scores on their communication rubrics scored by SPs for the PBA. While the rubric scores suggested students used most of the graded communication elements, open-ended feedback provided by SPs tended to focus on the humanistic aspects of communication that were not formally assessed by the rubric. The integrated analysis suggests there was a positive association between high student empathy and high scores on the communication rubric. Studies of actual patients corroborate the importance that the SPs placed on the importance of empathy to a successful encounter. Higher levels of provider empathy have been associated with increases in standardized measures of patient experience and satisfaction with health services. ${ }^{34,35}$ Outside of health care, higher levels of employee empathy indirectly contribute to perceptions of service quality, a benefit that may have implications pertaining to patient satisfaction, service experience, and health outcomes. ${ }^{16-19}$

This study also highlights the potential for PBAs using SPs to evaluate communication skills, such as empathy, that can be difficult to capture with traditional check-list style evaluation rubrics. While SPs were instructed not to use the single-item empathy assessment on the communication rubric, SPs frequently provided written comments about their observations and feelings related to student empathy, or lack thereof. Ultimately, the SP and student having a discussion about how the SP authentically felt during the encounter may provide more benefit than a single-item assessment of empathy. ${ }^{36,37}$

The interpretive analysis of SPs comments showed variation in students' ability to engage in empathetic communication and clinical empathy. Employing aspects of clinical empathy during high stakes standardized patient encounters appears to be a high-level skill which students may only partially deliver. For example, based on written feedback, students appeared to more often use empathy to understand the patient's perspective and less often used that understanding to then guide a therapeutic plan. Lo empathy encounters lacked essential elements of clinical empathy, especially responding to, and making use of cues from the SP when making recommendations. 
While we did not use student demographic data in our analysis, other studies report that student pharmacists may exhibit different levels of empathy based on a number of baseline characteristics, such as gender, altruism, age, and prior health care experiences. ${ }^{3}$ Further, professional students may exhibit low levels of empathy due to the stressful nature of assessments, a fear of making mistakes, and when engaged in challenging or difficult clinical scenarios. ${ }^{38,39}$ As such, there is need to explore factors that may be contributing to student pharmacist difficulty in developing clinical empathy and demonstrating their mastery within standardized patient encounters.

Given the importance of clinical empathy in patient encounters and the multitude of barriers that prevent students from employing clinical empathy, there are a number of recommendations that colleges and schools of pharmacy can consider: helping students understand how clinical empathy relates to the process of providing patient care, encouraging students to use clinical empathy within skills assessments, and assessing students more consistently on clinical empathy abilities.

First, in order to increase the use of empathetic communication and clinical empathy in high-stakes assessments, it may be important to use clinical empathy concepts to inform educational interventions and assessments within didactic coursework to help students make meaningful ties between clinical empathy and the Pharmacists' Patient Care Process (PPCP). ${ }^{40}$ This process, proposed by the Joint Commission of Pharmacy Practitioners, is a wholistic model of pharmaceutical care with a focus on patient-centricity. The process focuses on five steps to patient care: Collect, Assess, Plan, Implement, and Follow-Up. To help students value, develop, and employ clinical empathy skills, educational interventions should emphasize the importance of empathy throughout this process, using clinical empathy to guide patient-centered collection of data, assessment, and developing the clinical plan. ${ }^{41,42}$

Second, providing students with more low-stakes opportunities to demonstrate and develop clinical empathy skills; putting increased emphasis on the importance of clinical empathy within PBAs may encourage student pharmacists to use clinical empathy when engaging patients and making clinical recommendations that reflect a patient-centered approach. Making the demonstration of clinical empathy a required element of PBAs may encourage students to look for opportunities to use clinical empathy when it is their turn to be assessed.

Finally, to assure that student assessments are accurate representations of their clinical empathy abilities, formal rubrics and SP training should emphasize all three components of clinical empathy: listening, communicating, and developing a patient-centered clinical plan. To do so, it is worth considering the effectiveness of rubrics and SP instructional tools when evaluating clinical empathy, with additional consideration focused on innovative methods to assess clinical empathy and communication skills within PBAs. Open-ended SP comments could still be used alongside the ratings to provide the student with authentic feedback.

This study was subject to several limitations. This analysis used two cohorts of third-year student pharmacists at a single school of pharmacy which limits generalizability and the ability to assess empathy development across student cohorts and institutions. Additionally, the authors were limited to PBA rubrics with written feedback provided by SPs. Some communication rubrics did not have feedback comments, or the comments were inaccessible as some comments may have been written on the back of rubrics and lost to the archiving process or communicated only verbally to students, potentially altering the analysis. Further, the clinical scenario and/or SP characteristics may have influenced SP assessment of communication and empathy. While rank ordering yielded a statistically significant result, many of the scores were similar and did not exhibit normal distribution, suggesting a potential ceiling effect.

There are multiple opportunities for future studies that may expand assessment of student empathy ability within PBAs. Future studies should explore how the evaluator rubric used for student pharmacist communication assessment compares to existing patient-provider communication frameworks and tools, such as The Four Habits Model and PatientCentered Communication Tools (PaCT) ${ }^{43,44}$ Given that ordinal data was unavailable for empathy assessment, future studies should explore how single-item empathy scores compare to qualitative responses. Additionally, observational methods using video and validated communication coding schemes should be considered in future studies instead of, or in addition to, SP comments. While not modeled in the present study, an important area for future work is to consider how implicit biases related to ethnicity, gender, or other characteristics may affect SP evaluations of students as these factors have been shown to influence learner self-evaluations and patient evaluations of empathy ${ }^{30,45}$ More work is needed to evaluate clinical empathy skills longitudinally, specifically how student pharmacist's clinical empathy skills are affected by ongoing clinical education and the effect of empathy on patient experience with pharmacy services. Additional work should focus on differences in empathy based on SP and student pharmacist pairing, as well as considering additional empathy-related interventions to help students develop and employ clinical empathy in patient encounters, including but not limited to: mindfulness-based interventions ${ }^{46}$, professional development courses $^{3}$, and additional simulated clinical experiences. ${ }^{22-25}$ With increased focus on the importance and value of clinical empathy within pharmacy consultations ${ }^{10}$, 
additional research exploring how clinical empathy can be taught and measured within high-stakes assessment is needed. $^{47,48}$

\section{CONCLUSION}

Open-ended descriptions of student empathy provided by SPs were positively associated with high scores on a PBA communication rubric not formally assessing empathy. The majority of SP comments indicated the students had mixed use of empathy with many students struggling to consistently exhibit empathetic communication throughout the simulated encounter. Colleges and schools of pharmacy should consider methods to teach and assess clinical empathy skills, encouraging students to employ these skills during PBAs and beyond. With empathetic communication and clinical empathy integral to the patient encounter, exploring alternative tools for education and assessment may be useful in helping students acquire these skills.

\section{REFERENCES}

1. Jubraj B, Barnett NL, Grimes L, Varia S, Chater A, Auyeung V. Why we should understand the patient experience: clinical empathy and medicines optimisation. Int J Pharm Pract. 2016;24(5):367-370. doi:10.1111/ijpp.12268

2. Tamayo CA, Rizkalla MN, Henderson KK. Cognitive, Behavioral and Emotional Empathy in Pharmacy Students: Targeting Programs for Curriculum Modification. Original Research. Frontiers in Pharmacology. 2016-April-19 2016;7(96)doi:10.3389/fphar.2016.00096

3. Reed BN, Haines ST, Holmes ER. Can Empathy Be Learned? An Exploratory Analysis at Two Schools of Pharmacy. Am J Pharm Educ. 2020:ajpe8083. doi:10.5688/ajpe8083

4. Meyer-Junco L. Empathy and the new practitioner. American Journal of Health-System Pharmacy. 2015;72(23):2042-2058.

5. Fjortoft N, Van Winkle LJ, Hojat M. Measuring empathy in pharmacy students. Am J Pharm Educ. 2011;75(6):109-109. doi:10.5688/ajpe756109

6. LILJA J, LARSSON S, HAMILTON D, ISSAKAINEN J. Empathy as a communication strategy in the pharmacy — a study based on cognitive and behavioural analysis. Int J Pharm Pract. 2000;8(3):176-187. doi:https://doi.org/10.1111/j.2042-7174.2000.tb01003.x

7. Hojat M. Ten approaches for enhancing empathy in health and human services cultures. J Health Hum Serv Adm. Spring 2009;31(4):412-50.

8. $\quad$ Hojat M. Empathy in Patient Care: Antecedents, Development, Measurement, and Outcomes. Springer.; 2007.

9. Adams R. Clinical empathy: A discussion on its benefits for practitioners, students of medicine and patients. Journal of Herbal Medicine. 2012/06/01/ 2012;2(2):52-57.

doi:https://doi.org/10.1016/j.hermed.2012.04.004

10. Jubraj B, Barnett NL, Grimes L, Varia S, Chater A, Auyeung V. Why we should understand the patient experience: clinical empathy and medicines optimisation. Int J Pharm Pract. Oct 2016;24(5):367-70. doi:10.1111/ijpp.12268

11. Mercer SW, Reynolds WJ. Empathy and quality of care. Br J Gen Pract. Oct 2002;52 Suppl(Suppl):S912.

12. Halpern J. What is clinical empathy? J Gen Intern Med. Aug 2003;18(8):670-4. doi:10.1046/j.15251497.2003.21017.x

13. Hojat M, Gonnella JS, Nasca TJ, Mangione S, Vergare M, Magee M. Physician empathy: definition, components, measurement, and relationship to gender and specialty. American Journal of Psychiatry. 2002;159(9):1563-1569.

14. Riess H. The Science of Empathy. Journal of Patient Experience. 05/09 2017;4:237437351769926. doi:10.1177/2374373517699267

15. Riess H, Kelley JM, Bailey RW, Dunn EJ, Phillips M. Empathy training for resident physicians: a randomized controlled trial of a neuroscience-informed curriculum. J Gen Intern Med. Oct 2012;27(10):1280-6. doi:10.1007/s11606-012-2063-z 
16. Price S, Mercer SW, MacPherson H. Practitioner empathy, patient enablement and health outcomes: a prospective study of acupuncture patients. Patient education and counseling. 2006;63(1-2):239-245.

17. Mercer SW, Howie JG. CQI-2 - a new measure of holistic interpersonal care in primary care consultations. Br J Gen Pract. 2006;56(525):262-268.

18. Bikker AP, Mercer SW, Reilly D. A pilot prospective study on the consultation and relational empathy, patient enablement, and health changes over 12 months in patients going to the Glasgow Homoeopathic Hospital. Journal of Alternative \& Complementary Medicine. 2005;11(4):591-600.

19. Howick J, Moscrop A, Mebius A, et al. Effects of empathic and positive communication in healthcare consultations: a systematic review and meta-analysis. J R Soc Med. Jul 2018;111(7):240-252. doi:10.1177/0141076818769477

20. Bas-Sarmiento P, Fernández-Gutiérrez M, Baena-Baños M, Correro-Bermejo A, Soler-Martins PS, de la Torre-Moyano S. Empathy training in health sciences: A systematic review. Nurse Education in Practice. 2020/03/01/ 2020;44:102739. doi:https://doi.org/10.1016/j.nepr.2020.102739

21. Levett-Jones T, Cant R, Lapkin S. A systematic review of the effectiveness of empathy education for undergraduate nursing students. Nurse Educ Today. Apr 2019;75:80-94. doi:10.1016/j.nedt.2019.01.006 22. Sjoquist LK, Cailor S, Conkey L, Wilcox R, Ng B, Laswell EM. A simulated patient experience to improve pharmacy student empathy for patients on hemodialysis. Curr Pharm Teach Learn. 2020/07/01/ 2020;12(7):827-833. doi:https://doi.org/10.1016/j.cpt1.2020.02.018

23. Silvia RJ. A Music Assignment to Develop Pharmacy Students' Empathy Toward People with Opioid Use Disorder. Am J Pharm Educ. 2020;84(4):7631. doi:10.5688/ajpe7631

24. Abeyaratne C, Bell JS, Dean L, White P, Maher-Sturgess S. Engaging older people as university-based instructors: A model to improve the empathy and attitudes of pharmacists in training. Curr Pharm Teach Learn. 2020/01/01/2020;12(1):58-64. doi:https://doi.org/10.1016/j.cptl.2019.10.011

25. Underwood M, Ronald K. A pilot study assessing the impact of a polypharmacy mock medication simulation on student adherence and empathy. Curr Pharm Teach Learn. 2020/08/01/ 2020;12(8):956-962. doi:https://doi.org/10.1016/j.cptl.2020.04.020

26. Laughey W, Sangvik Grandal N, Stockbridge C, Finn GM. Twelve tips for teaching empathy using simulated patients. Medical Teacher. 2019/08/03 2019;41(8):883-887. doi:10.1080/0142159X.2018.1481283 27. Laughey W, Sangvik Grandal N, M Finn G. Medical communication: the views of simulated patients. Medical Education. 2018;52(6):664-676.

28. Chen JY, Chin W-Y, Tsang JPY. How clinician examiners compare with simulated patients in assessing medical student empathy in a clinical exam setting. Medical Teacher. 2020/01/02 2020;42(1):86-91. doi:10.1080/0142159X.2019.1665635

29. Cunico L, Sartori R, Marognolli O, Meneghini AM. Developing empathy in nursing students: a cohort longitudinal study. J Clin Nurs. Jul 2012;21(13-14):2016-25. doi:10.1111/j.1365-2702.2012.04105.x 30. Berg K, Blatt B, Lopreiato J, et al. Standardized patient assessment of medical student empathy: ethnicity and gender effects in a multi-institutional study. Acad Med. Jan 2015;90(1):105-11. doi:10.1097/acm.0000000000000529

31. Nunes P, Williams S, Sa B, Stevenson K. A study of empathy decline in students from five health disciplines during their first year of training. International Journal of Medical Education. 02/08 2011;2doi:10.5116/ijme.4d47.ddb0

32. Hojat M, Vergare MJ, Maxwell K, et al. The devil is in the third year: a longitudinal study of erosion of empathy in medical school. Acad Med. Sep 2009;84(9):1182-91. doi:10.1097/ACM.0b013e3181b17e55

33. Barnett SG, Porter AL, Allen SM, et al. Expert Consensus to Finalize a Universal Evaluator Rubric to Assess Pharmacy Students' Patient Communication Skills. Am J Pharm Educ. 2020;84(12):848016. doi:10.5688/ajpe848016

34. Chaitoff A, Sun B, Windover A, et al. Associations Between Physician Empathy, Physician Characteristics, and Standardized Measures of Patient Experience. Academic Medicine. 2017;92(10) 
35. Walsh S, O’Neill A, Hannigan A, Harmon D. Patient-rated physician empathy and patient satisfaction during pain clinic consultations. Irish Journal of Medical Science (1971 -). 2019/11/01 2019;188(4):1379-1384. doi:10.1007/s11845-019-01999-5

36. Bokken L, Rethans J-J, Jöbsis Q, Duvivier R, Scherpbier A, van der Vleuten C. Instructiveness of Real Patients and Simulated Patients in Undergraduate Medical Education: A Randomized Experiment. Academic Medicine. 2010;85(1):148-154. doi:10.1097/ACM.0b013e3181c48130

37. Bokken L, Rethans J-J, Scherpbier AJJA, van der Vleuten CPM. Strengths and Weaknesses of Simulated and Real Patients in the Teaching of Skills to Medical Students: A Review. Simulation in Healthcare. 2008;3(3):161-169. doi:10.1097/SIH.0b013e318182fc56

38. Ward J. The Empathy Enigma: Does It Still Exist? Comparison of Empathy Using Students and Standardized Actors. Nurse Educ. May-Jun 2016;41(3):134-8. doi:10.1097/nne.0000000000000236

39. Ward J, Cody J, Schaal M, Hojat M. The empathy enigma: an empirical study of decline in empathy among undergraduate nursing students. J Prof Nurs. Jan-Feb 2012;28(1):34-40.

doi:10.1016/j.profnurs.2011.10.007

40. Practitioners JCoP. Pharmacists' Patient Care Process 2014. May 29th, 2014. https://jcpp.net/wpcontent/uploads/2016/03/PatientCareProcess-with-supporting-organizations.pdf

41. Vyas D, Chen M, Boyce EG, Galal SM, Rogan EL, Maker J. Training students on the Pharmacist

Patient Care Process using an electronic health record and simulations. Pharmacy Education. 2019;19:374-380.

42. Whittaker CF, Nelson SA, Tom SE. Impact of the Pharmacists' Patient Care Process on Medication Adherence in Older Adults with Multimorbidity. The Senior care pharmacist. 2019;34(7):456-463.

43. Grice GR, Gattas NM, Prosser T, et al. Design and Validation of Patient-Centered Communication Tools (PaCT) to Measure Students' Communication Skills. Am J Pharm Educ. 2017;81(8):5927. doi:10.5688/ajpe5927

44. Naughton CA. Patient-Centered Communication. Pharmacy (Basel). Feb 13 2018;6(1)doi:10.3390/pharmacy6010018

45. Roberts BW, Trzeciak CJ, Puri NK, Mazzarelli AJ, Trzeciak S. Racial and socioeconomic disparities in patient experience of clinician empathy: a protocol for systematic review and meta-analysis. BMJ Open. 2020;10(6):e034247. doi:10.1136/bmjopen-2019-034247

46. Witry MJ, Murry LT, Ray ME. Considerations for the incorporation of mindfulness into pharmacy education. Currents in Pharmacy Teaching and Learning. 2020/03/01/ 2020;12(3):247-250. doi:https://doi.org/10.1016/j.cptl.2019.12.007

47. Cavaco A, Roter D. Pharmaceutical consultations in community pharmacies: utility of the Roter Interaction Analysis System to study pharmacist-patient communication. Int J Pharm Pract. Jun 2010;18(3):141-8.

48. Kubota Y, Yano Y, Seki S, et al. Assessment of pharmacy students' communication competence using the Roter Interaction Analysis System during objective structured clinical examinations. Am J Pharm Educ. Apr 11 2011;75(3):43. doi:10.5688/ajpe75343 
Figure 1. Mixed Methods Study Design

PBA Communication

Rubrics $(n=141)$

QUANT Item Scores (20 items)

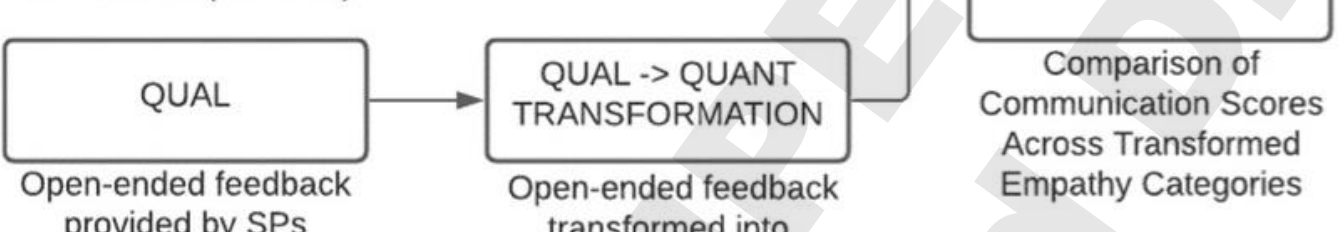

provided by SPS transformed into

empathy categories 
Table 1. Empathy Categorization and Representative Quotes

\begin{tabular}{ll}
\hline $\begin{array}{l}\text { Empathy } \\
\text { Category }\end{array}$ & Additional Quote Examples \\
\hline Low & "I feel at a disadvantage and maybe uncomfortable when I don't follow the meaning [of a word] ... I even \\
Empathy & feel stupid or inadequate. Many words mean something very different to me then to you." \\
& "Student was looking down at... about half of the time, so I felt a disconnect." \\
& "I informed you I had done all I was going to do about my diet and exercise. However, you were critical \\
& about that, said I should take even a short walk now and then. You began quizzing me why I didn't want to \\
& do any more myself...I got a bit frustrated with that and expressed some anger at that point."
\end{tabular}

Mixed "Slowing the pace and allowing more opportunity for the patient to reflect and formulate questions and Empathy concerns."

"...not rushed, I think there is room to slow down a bit. It will help give you time to think."

“... several times. Looked down at notes and not look up at when speaking to me...”

High "The line they used "Your concerns are my concerns" left me feeling important, validated, and Empathy understood.”

"Nice job with empathy-when I mentioned being on 4 medications you immediately told me you understood, that must be hard, etc. Non-judgmental-when I told you about forgetting bedtime med you said that is understandable and then said we could do something to fix that. Also, understanding when I mentioned not being able to improve my diet-you emphasized how good small changes are."

“Student had fantastic empathy, communication, and nonverbal communication skills.”

“...nodding their head which helped me feel heard."

"Student displayed strong concern for my well-being...I felt very engaged with the eye contact and smile, no judging..."

"... aware of how the patient is responding and accommodating to their needs and that is what is important."

"The student and I worked together before and it is such a pleasure to see how polished, empathetic, and thorough [they] were in the encounter."

"You are aware of how the patient is responding and accommodating to their needs and that is what is important. The interview was very short, but you were organized and efficient and I never felt that you were rushing through it.", 
Table 2. Descriptive statistics for communication scores across empathy categories

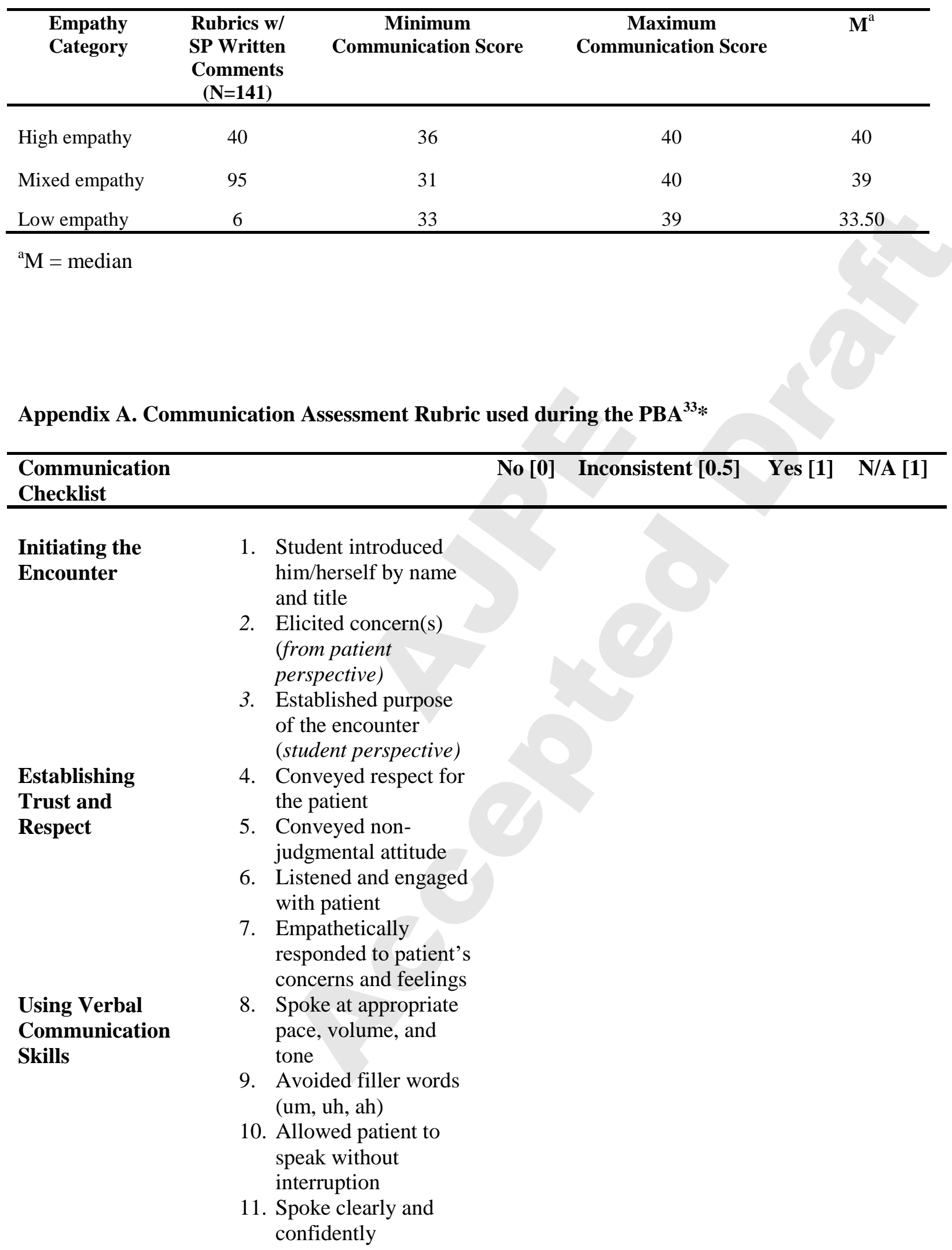


12. Used patient friendly language

Using Non-

Verbal

13. Made appropriate eye

Communication

Skills contact

14. Displayed appropriate body language

Managing the

Encounter

15. Completed one topic prior to moving to the next

16. Utilized time efficiently

17. Provided opportunity for and responded to questions

Concluding the

18. Used teach-back

19. Achieved mutual agreement with the plan

20. Provides closure to the encounter 